\title{
Temperature field simulation and phantom validation of a Two-armed Spiral Antenna for microwave thermotherapy
}

\author{
Yongxing Du ${ }^{\mathrm{a}}$, Lingze Zhang ${ }^{\mathrm{b}}$, Lulu Sang ${ }^{\mathrm{a}}$ and Daocheng $\mathrm{Wu}^{\mathrm{b}, *}$ \\ anner Mongolia University of Science and Technology, Baotou, Mongolia, China \\ ${ }^{\mathrm{b}} X i$ 'an Jiaotong University, Xi'an, Shaanxi, China
}

\begin{abstract}
In this paper, an Archimedean planar spiral antenna for the application of thermotherapy was designed. This type of antenna was chosen for its compact structure, flexible application and wide heating area. The temperature field generated by the use of this Two-armed Spiral Antenna in a muscle-equivalent phantom was simulated and subsequently validated by experimentation. First, the specific absorption rate (SAR) of the field was calculated using the Finite Element Method (FEM) by Ansoft's High Frequency Structure Simulation (HFSS). Then, the temperature elevation in the phantom was simulated by an explicit finite difference approximation of the bioheat equation (BHE). The temperature distribution was then validated by a phantom heating experiment. The results showed that this antenna had a good heating ability and a wide heating area. A comparison between the calculation and the measurement showed a fair agreement in the temperature elevation. The validated model could be applied for the analysis of electromagnetic-temperature distribution in phantoms during the process of antenna design or thermotherapy experimentation.
\end{abstract}

Keywords: Archimedean planar spiral antenna, finite-difference-Bioheat equation (FD-BHE) methods, microwave thermotherapy, phantom

\section{Introduction}

Hyperthermia $\left(42^{\circ} \mathrm{C}\right.$ to $\left.46^{\circ} \mathrm{C}\right)$ is an effective method of treatment of tumors. The method involves the application of heat in order to destroy tumor cells without damage to normal tissues. It has been shown that a sustained temperature above $42^{\circ} \mathrm{C}$ will cause necrosis of living cells [1-4]. To date, several methods have been developed to successfully induce temperature rises either locally in selected regions of specific organs or over the whole body. Since microwave (MW) thermotherapy has the advantage of providing a comfortable and painless treatment environment for patients, it has been used in medicine for cancer treatment and the treatment of other diseases since early eighties [5,6]. Researchers have designed many types of radiators that are suitable for therapy on superficial tumors [7-9].

Designs of ablation antennas are often tested through in vivo or in vitro experimental studies [10]. However, the former requires animal experimentation, and the latter lacks biological reality [11]. Furthermore, the experimental approach can only verify the performance of the antenna after the antenna has been constructed, which makes the design complex and costly. This problem has encouraged researchers

\footnotetext{
${ }^{*}$ Corresponding author: Daocheng Wu, Xi' an Jiaotong University, Xi'an, Shaanxi, China. E-mail: wudaocheng@mail.xjtu. edu.cn.
}

0928-7329/16/\$35.00 @ 2016 - IOS Press and the authors. All rights reserved

This article is published online with Open Access and distributed under the terms of the Creative Commons Attribution NonCommercial License. 


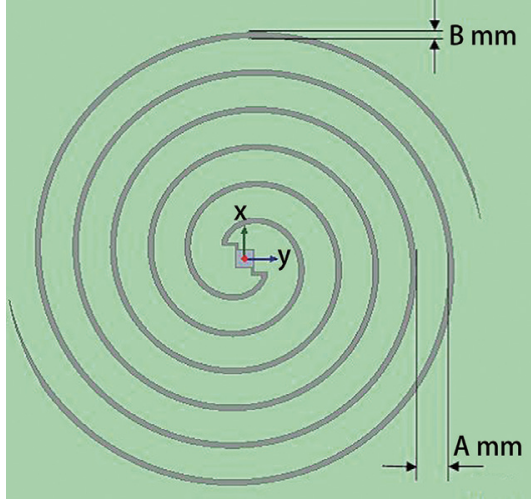

(a)

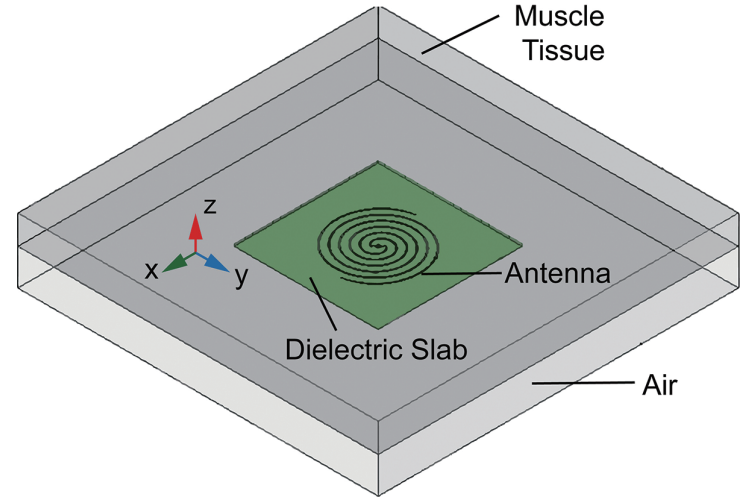

(b)

Fig. 1. (a) The basic structure of the spiral antenna. (b) The model used for calculating electromagnetic field and temperature field data.

to develop a numerical tool to solve the electromagnetic and thermal problem inside the tissue [11-13]. Bernardi et al. (2004) calculated the absorbed electromagnetic power (SAR) and temperature elevation in a phantom with the finite-difference time-domain (FDTD) method [11]. In that study, a cap-choke antenna was embedded in the phantom and fiber-optic temperature probes were placed at different radial distances from the antenna to observe axial temperature distributions.

In this paper, an Archimedean planar spiral antenna was chosen for design and study in simulation and experiment because of its compact structure, flexible application and wide heating area. Unlike the cap-choke antenna, this antenna is mainly used for therapy on superficial tumors. The SAR and temperature elevation were investigated for a phantom of the antenna. The antenna and phantom were modeled, and the SAR using FEM by Ansoft's HFSS was calculated. With the calculated SAR, the temperature elevation in the phantom of this antenna was calculated using an explicit FD approximation of the bioheat equation (BHE) which was similar to the previous numerical-experimental study [11]. The calculation model of the electromagnetic-temperature distribution was then validated by a phantom experiment.

\section{Methods}

\subsection{The simulation model of the antenna}

The simulation model consisted of a muscle tissue-like phantom and an antenna with an air gap (Fig. 1(b)). The antenna was based on a $50 \Omega$ semirigid copper-Teflon (dielectric constant of Teflon $\varepsilon=2.1$ ) coaxial cable with inner and outer diameters of $0.9 \mathrm{~mm}$ and $3 \mathrm{~mm}$ respectively. The inner copper connects to the Archimedean spiral antenna. As shown in Fig. 1(a), A is the distance between the spiral circles, $\mathrm{B}$ is the spiral wire's width, and $\mathrm{N}$ is the circle numbers of the spiral antenna. After optimizing the parameter, we obtain $\mathrm{A}=3.3 \mathrm{~mm}, \mathrm{~B}=5.4 \mathrm{~mm}$ and $\mathrm{N}=3$. The tissue-like phantom area was a box that measured $100 * 100 * 20 \mathrm{~mm}^{3}$. The dielectric constant and conductivity of the phantom were based on previous research from [14]. The air gap between the phantom and the antenna was 0.5 $\mathrm{mm}$. The power and the frequency of the MW were set to $10 \mathrm{~W}$ and $2450 \mathrm{MHz}$ respectively. 


\subsection{SAR and temperature distribution computation}

Numerical study was performed using the FEM by Ansoft's HFSS to calculate electromagnetic field and an FD solution of the bioheat equation (BHE) to calculate temperature field.

The stabilization time of the electromagnetic field is quite short compared to the temperature field. The dielectric constant and conductivity of the phantom also do not change significantly as the temperature increases. Therefore, the electromagnetic field and temperature field can be calculated separately.

The SAR data of the field was calculated as $\operatorname{SAR}(W / K g)=\sigma / 2 \rho \cdot|E|^{2}$, where $\rho$ and $C$ are, respectively, the density and specific heat of the tissue, and $E$ is the electric field within the tissue.

The temperature distribution in the tissue follows the well-known bioheat equation [15]

$$
\rho \cdot C \frac{\partial T}{\partial t}=\nabla \cdot(K \nabla T)+A+\rho \cdot S A R+b\left(T-T_{b}\right)
$$

with the boundary condition

$$
-K\left(\frac{\partial T}{\partial n}\right)=h\left(T-T_{a}\right)
$$

where $\rho$ and $C$ are, respectively, the density and specific heat of the tissue, is the temperature at time $t, K$ is the thermal conductivity, $A$ is metabolic heat production of the tissue, $b$ accounts for blood perfusion, $T_{b}$ is the blood temperature, $T_{a}$ is the ambient temperature, $n$ is the unit vector normal to the surfaces of the phantom, and $h$ is the convective heat-transfer coefficient. As the ex vivo situation (phantom) is considered, both metabolic heat and blood perfusion are neglected. The equation in Eq. (1) can be simplified to

$$
\rho \cdot C \frac{\partial T}{\partial t}=\nabla \cdot(K \nabla T)+\rho \cdot S A R
$$

Because the FDTD method was used for the temperature field computation, Eqs (1) and (2) were expanded in their finite difference approximations.

$$
\begin{array}{r}
T^{n+1}(i, j, k)=T^{n}(i, j, k)+\frac{\Delta t \cdot \sigma(i, j, k)}{2 C(i, j, k) \cdot \rho(i, j, k)} \cdot|E|^{2}+ \\
\frac{\Delta t \cdot K(i, j, k)}{C(i, j, k) \cdot \rho(i, j, k)} \cdot\left[\begin{array}{l}
\frac{T^{n}(i+1, j, k)+T^{n}(i-1, j, k)-2 T^{n}(i, j, k)}{\Delta x^{2}}+ \\
\frac{T^{n}(i, j+1, k)+T^{n}(i, j-1, k)-2 T^{n}(i, j, k)}{\Delta y^{2}}+ \\
\frac{T^{n}(i, j, k+1)+T^{n}(i, j, k-1)-2 T^{n}(i, j, k)}{\Delta z^{2}}
\end{array}\right] \\
-K \frac{T^{n}(i+1, j, k)-T^{n}(i, j, k)}{\Delta x}=h\left(T^{n}(i, j, k)-T_{a}\right)
\end{array}
$$

In order to ensure numerical stability, $t$ was chosen to satisfy

$$
t \leqslant \frac{\rho C \delta^{2}}{6 K}
$$




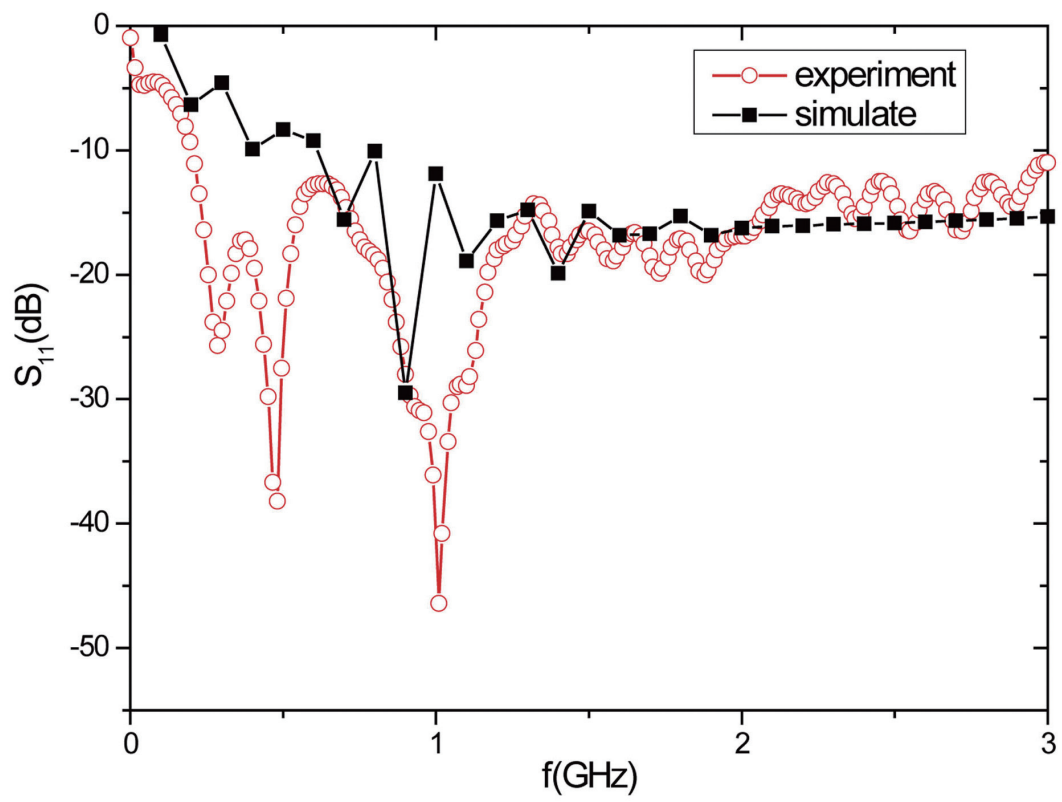

Fig. 2. The reflection coefficient of the microstrip Archimedean spiral antenna, comparing numerical with experimental results.

\subsection{Phantom heating experiment}

To conduct the experiment, the designed antenna was manufactured. In the experiment, the antenna was connected to a MW generator by a semirigid copper-Teflon coaxial cable. The frequency and power of the MW were set to $2450 \mathrm{MHz}$ and $10 \mathrm{~W}$ respectively. A phantom with dimensions of $50 \mathrm{~mm} * 50 \mathrm{~mm}$ * $18 \mathrm{~mm}$ was prepared. The phantom was composed of a mixture of $8.45 \% \mathrm{TX}-150,15.2 \%$ polyethylene powder, $75.45 \%$ water, and $0.9 \% \mathrm{NaCl}$, which is considered muscle-equivalent material [14]. To display the temperature distribution, $0.5 \%$ thermochromic material was added when preparing the phantom. The phantom was placed above the antenna, with a piece of plastic film between the two as an insulator. The MW was applied to heat the phantom for $10 \mathrm{~min}$, and photos were taken immediately after heating.

\section{Results and discussion}

A microstrip Archimedes planar spiral antenna was manufactured. The reflection coefficient of the microstrip Archimedes spiral antenna was measured by Angilent E4438C RF Integrative Vector Network Analyzer. The result shows that the applicator can work at a broadband (see Fig. 2). The parameter S11 of the experimental result and the numerical result were $-15.3 \mathrm{~dB}$ and $-15.8 \mathrm{~dB}$ at $2.45 \mathrm{GHz}$. The comparison shows good agreement between the calculated and experimental reflection coefficients.

The antenna showed good heating ability over a wide heating area (shown in Fig. 2(a)(d)). Temperature elevation occurred in the shape of a semi elliptical sphere. The two boundaries of the three different colors in the phantom represent the temperatures $37^{\circ} \mathrm{C}$ and $42^{\circ} \mathrm{C}$. A wide area was heated to more than $42^{\circ} \mathrm{C}$. Although the hottest place was above $80^{\circ} \mathrm{C}$ in the middle of the surface, generally the excess energy can be carried away by a water cooling system or by intermittent heating. Because the antenna was designed to treat superficial tumors, with a high frequency of $2450 \mathrm{MHz}$, the electromagnetic energy penetrated shallowly. 


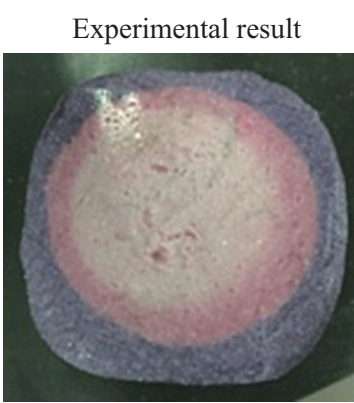

(a)

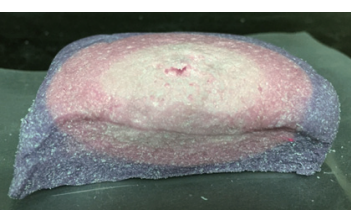

(d)

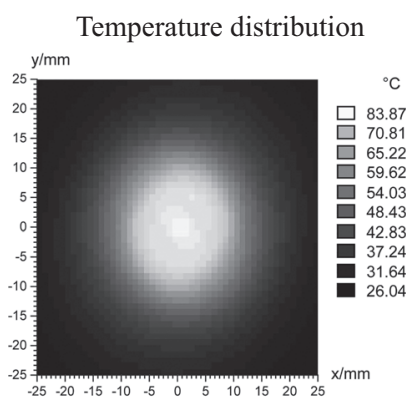

(b)

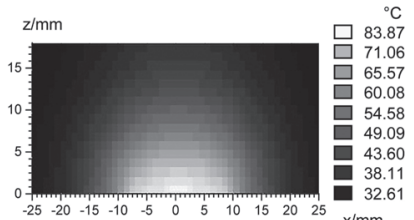

(e)

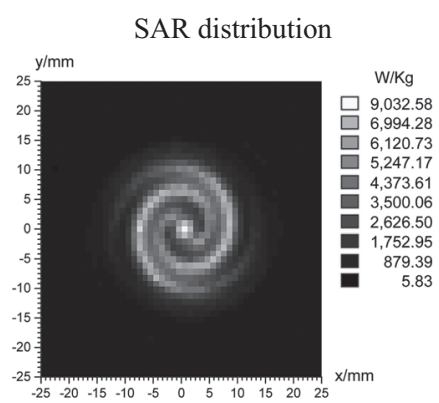

(c)

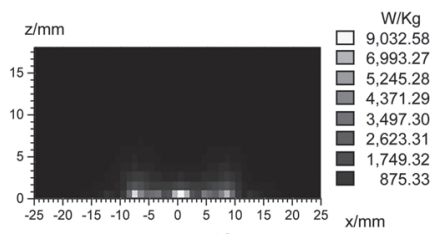

(f)

Fig. 3. (a), (b) and (c) show the computation results of the SAR and temperature distribution at section $z=0$ and the corresponding experimental result. Figures 3(d), (e) and (f) show the computation result of the SAR and temperature distribution at section $y=0$ and the corresponding experimental result.

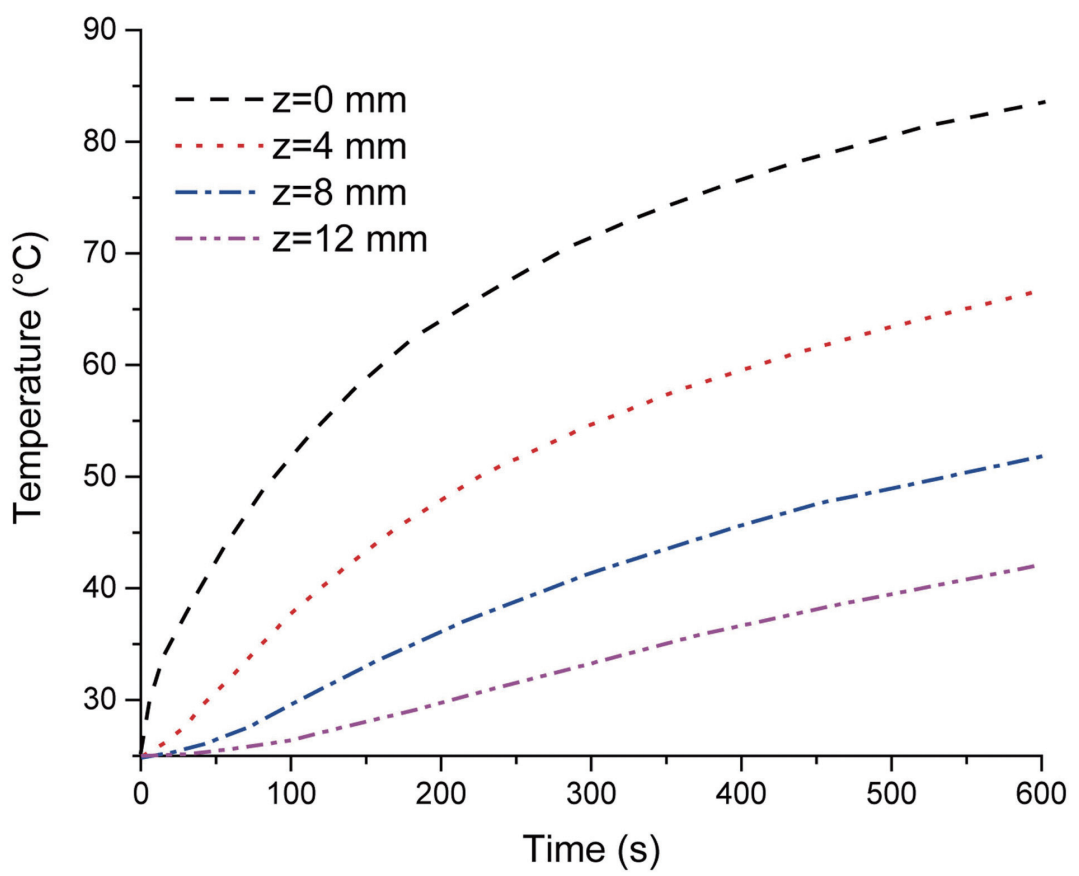

Fig. 4. Time course of temperature inside the phantom at section $y=0$ for four different depths $(0,4,8$, and $12 \mathrm{~mm}$ from section $z=0$ ).

When Figs 3(a)(d) is compared to Figs 3(b)(e), it is clear that the simulation of the temperature distribution was consistent with the experimental result. According to these results, it can be projected that 


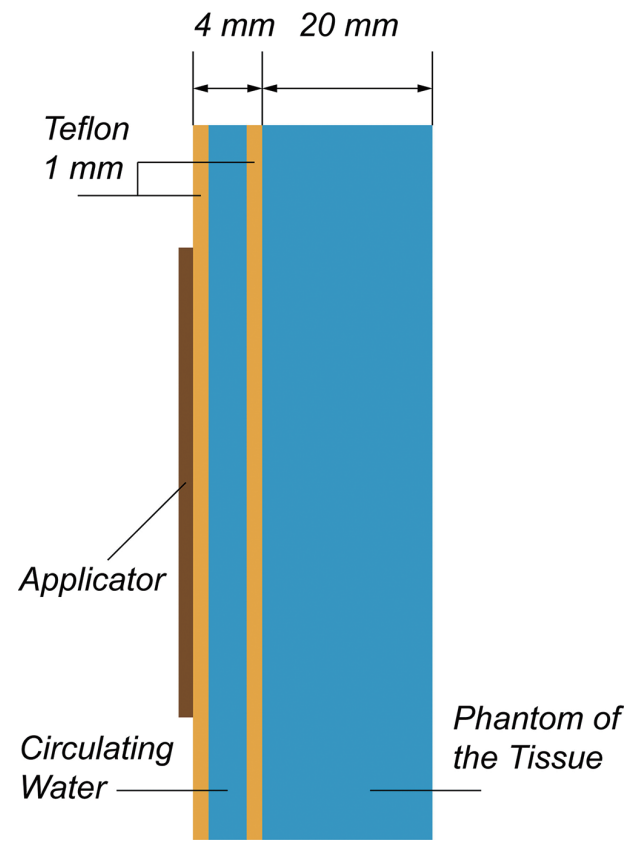

Fig. 5. Water cooling system.

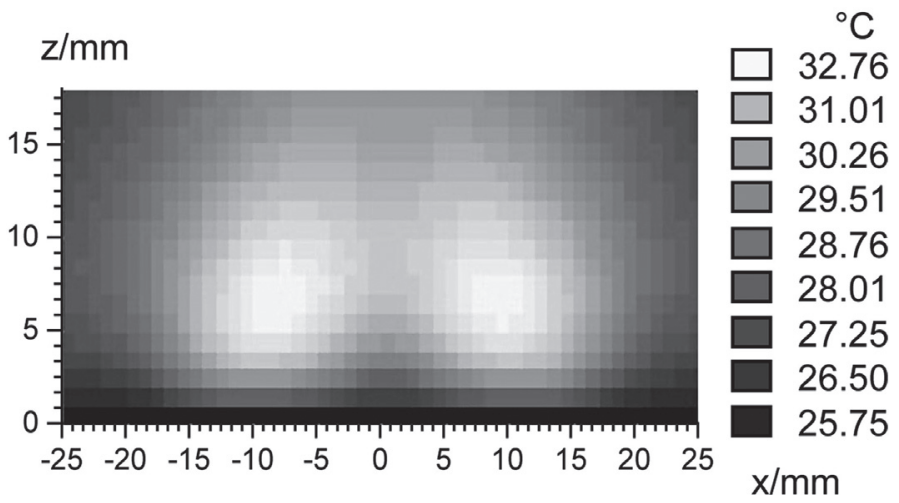

Fig. 6. Temperature distribution at $y=0 \mathrm{~mm}$ of the phantom.

the FD-BHE calculations used in this study can be effectively applied to real situations. Other antennas can potentially be simulated in the same way.

It can be seen from Figs 3(b)(c) and (e)(f) that the SAR distribution is quite different from the temperature distribution. This indicates the importance of calculating not only the SAR distribution but also the temperature distribution when antennas are designed for thermotherapy.

The computed time courses of temperature increments for five depths within the phantom at section $y=0$ are shown in Fig. 4. The curves refer to the first $10 \mathrm{~min}$ of the MW application. The temperature field had not yet reached a steady state at $10 \mathrm{~min}$. At $10 \mathrm{~min}$, at $z=12 \mathrm{~mm}$ section, the temperature reached $42^{\circ} \mathrm{C}$.

These results show that the superficial temperature can reach over $60^{\circ} \mathrm{C}$ without the cooling medium, which can cause burns to the skin. With the validated model, it is possible to calculate the temperature 
filed with a water cooling system (see Fig. 5). Figure 6 shows the predicted temperature distribution when the water of $25^{\circ} \mathrm{C}$ is circulated to cool down the surface of the skin. The power and the frequency of microwave were set to $10 \mathrm{~W}$ and $2450 \mathrm{MHz}$ respectively, as they were in the previous experiment. The numerical result shows that a cooling medium is necessary to prevent skin burns.

\section{Conclusion}

In this paper, a compact Archimedean planar spiral antenna with wide heating area was designed, and its performance was investigated both numerically and experimentally. Simulation of the electromagnetic power absorption and the temperature increase were carried out using the FEM and FDTD methods, respectively. When the calculation and the measurement were compared, a fair agreement was shown in the temperature elevations. Meanwhile, the difference between the SAR distribution and the temperature distribution indicated that temperature field should be considered in addition to SAR distribution in the design of an antenna. In future research, this simulation model will aid in the designs of new antennas and hyperthermia treatments for MW thermotherapy.

\section{Acknowledgments}

The authors would like to thank the National Natural Science Foundation $(61301073,61501266)$ and The Inner Mongolia Natural Science Foundation (2015MS0624) for support during the development.

\section{References}

[1] R. Cavaliere, E.C. Ciocatto, B.C. Gionanella, C. Heidelberger, R.O. Johnson, M. Margottini, B. Mondovi, G. Moricca, and A. Rossi-Fanelli, Selective heat sensitivity of cancer cells biochemical and clinical studies, Cancer, vol. 20, pp. 1351-1381, 1967.

[2] J.E. Robinson, M.J. Wizenberg, and W.A. McCready, Combined hyperthermia and radiation, an alternative to heavy particle therapy for reduced oxygen enhancement ratios, Nature, vol. 251, pp. 521-522, 1974.

[3] R.A. Steeves, Hyperthermia in cancer therapy: Where are we today and where are we going? Bull. NY Acad. Med., vol. 68, pp. 342-350, 1992.

[4] W. Dewey, Arrhenius relationships from the molecule and cell to the clinic, Int. J. Hyperthermia, vol. 10, pp. 457-483, 1994.

[5] K.H. Luk, R.M. Hulse, and T.L. Phillips, Hyperthermia in Cancer Therapy, West J Med., 132(3), pp. 179-185, 1980.

[6] J. Vrba, L. Oppl, D. Vrba, J. Vorlíček, B. Vrbová, and D. Havelka, Prospective Applications of EM fields in Medicine, PIERS Proceedings, 2011.

[7] M.J. Ammann, S. Curto, X.L. Bao, and P. McEvoy, Antenna design considerations for high specific absorption rate in local hyperthermia treatment. Antennas and Propagation Society International Symposium, pp. 1-4, 2008.

[8] J. Šafař́k, O. Rychlík, Planar applicators for local hyperthermia. Radioelektronika 2009, 19th International Conference. pp. 349-352, 2009.

[9] D. Correia, H.P. Kok, M. de Greef, A. Bel, N. van Wieringen, and J. Crezee, Body Conformal Antennas for Superficial Hyperthermia: The Impact of Bending Contact Flexible Microstrip Applicators on Their Electromagnetic Behavior, IEEE Trans. Biomed. Eng., vol. 56, no. 12, pp. 2917-2926, 2009.

[10] A.J. Greenspon, Advances in catheter ablation for the treatment of cardiac arrhythmias, IEEE Trans. Microwave Theory Tech., vol. 48, pp. 2670-2675, Dec. 2000.

[11] P. Bernardi, M. Cavagnaro, J.C. Lin, S. Pisa, and E. Piuzzi, Distribution of SAR and Temperature Elevation Induced in a Phantom by a Microwave Cardiac Ablation Catheter, IEEE Trans. Microwave Theory Tech., vol. 52, pp. 1978-1986, 2004.

[12] M. Cavagnaro, C. Amabile, P. Bernardi, S. Pisa, and N. Tosoratti, A Minimally Invasive Antenna for Microwave Ablation Therapies: Design, Performances, and Experimental Assessment, IEEE Trans. Biomed. Eng., vol. 58, no. 4, pp. 949-959, 2011. 
[13] W.C. Choi, K.J. Kim, J. Kim, and Y.J. Yoon, Compact Microwave Radiator for Improving Heating Uniformity in Hyperthermia System, IEEE Antennas and Wireless Propagation Letters, vol. 13, pp. 1345-1348, 2014.

[14] A.W. GUY, Analysis of electromagnetic fields induced in biological tissues by thermographic studies on equivalent phantom models, IEEE Trans. Microwave Theory Tech., vol. MTT-19, pp. 205-214, Feb. 1971.

[15] H.H. Pennes, Analysis of tissue and arterial blood temperature in the resting human forearm, J. Appl. Physiol., vol. 1, pp. 93-122, 1948. 